Tôhoku Math. Journ. 39 (1987), 533-542.

\title{
BOUNDED PROJECTIONS ONTO HOLOMORPHIC HARDY SPACES ON PLANAR DOMAINS
}

\author{
Hitoshi ARAI* \\ (Received September 12, 1986)
}

1. Introduction. Throughout this paper, $D \subset C$ is a domain bounded by finitely many non-intersecting simple closed $C^{4}$ regular curves. We denote by $m_{0}$ the area Hausdorff measure on the boundary $\partial D$ of the domain $D$, and by $m_{1}$ and $m_{2}$ two different harmonic measures relative to $D$. The holomorphic Hardy spaces $H^{p}\left(m_{j}\right)$ on $\partial D$ are defined as the $L^{p}\left(m_{j}\right)$-norm closure of $A(\partial D) 1 \leqq p<\infty$, where $A(\partial D)$ is the class of continuous functions $f$ on $\partial D$ whose Poisson integral PI $[f]$ is analytic in $D$. This paper is concerned with projection operators of $L^{p}\left(m_{j}\right)$ onto $H^{p}\left(m_{j}\right)$.

As is well known, there are two bounded projection operators of $L^{2}\left(m_{j}\right)$ onto $H^{2}\left(m_{j}\right)$. One of them is the Cauchy projection $H$ and the other is the orthogonal projection $P_{j}$. These operators are useful to study real or holomorphic Hardy spaces. In particular, $H$ and $P_{0}$ also play important roles in the theory of partial differential equations and of conformal mappings. In addition, $P_{1}$ and $P_{2}$ are deeply related with uniform algebras.

In this paper, we show correlations between $H, P_{0}, P_{1}$ and $P_{2}$, and give some applications to holomorphic Hardy spaces. Our investigation is motivated by the following interesting theorem by Kerzman and Stein [10]:

THEOREM KS ([10]; see also [3]). Let $D$ be a bounded, simply connected $C^{\infty}$ domain in the plane, and $H^{*}$ be the adjoint of $H$ on the Hilbert space $L^{2}\left(m_{0}\right)$. Then:

(1) $H^{*}-H$ is an integral operator with a smooth kernel. Hence it is compact on $L^{2}\left(m_{0}\right)$.

(2) Further, $I-\left(H^{*}-H\right)$ is an injective bounded operator of $L^{2}\left(m_{0}\right)$ onto $L^{2}\left(m_{0}\right)$, and

$$
P_{0}=H\left(I-H^{*}+H\right)^{-1} .
$$

This result tells us a relation between $H$ and $P_{0}$. On the other hand,

* Partly supported by the Grants-in-Aid for Encouragement of Young Scientists, The Ministry of Education, Science and Culture, Japan. 
our main theorem stated later implies that $P_{1}$ also can be written in terms of $H$, and $P_{2}$ can be represented in terms of $H$ and $P_{1}$. Moreover, it gurantees that each $P_{j}$ is bounded on the Hardy space $H_{\max }^{1}$ in the sense of Fefferman and Stein.

Let the complement $D^{c}$ of $D$ have $n+1$ connected components. Denote by $G_{0}$ the unbounded component of $D^{c}$ and by $G_{\mu}$ for $\mu=1, \cdots, n$ the bounded components of $D^{c}$. For every $G_{k}(k=0, \cdots, n)$ let $l_{k}$ be the length of $\partial G_{k}$ and denote

$$
\partial G_{k}=\left\{\alpha_{k}(s): \sum_{d=0}^{k} l_{d-1} \leqq s<\sum_{d=0}^{k+1} l_{d-1}\right\},
$$

where $l_{-1}=0$ and $\alpha_{k}$ is a unit speed simple closed $C^{4}$ curve which surrounds $G_{k}$. We suppose that $\alpha_{0}$ is positively oriented and $\alpha_{1}, \cdots, \alpha_{n}$ are negatively oriented. For simplicity we use the notation

$$
\alpha(s)=\alpha_{k}(s), \quad \text { for } \quad s \in I_{k} \equiv\left[\sum_{d=0}^{k} l_{d-1}, \sum_{d=0}^{k+1} l_{d-1}\right),
$$

if there is no confusion.

Let $K(\cdot, \cdot)$ be the Cauchy kernel, that is,

$$
K(s, t)=D_{+} \alpha(t) /[\alpha(t)-\alpha(s)],
$$

for $(s, t) \in[0, L) \times[0, L)-\{$ diagonal $\}$, where $L=\sum_{d=0}^{n} l_{d}$, and $D_{+} F(t)=$ $\lim _{h \rightarrow+0}[F(t+h)-F(t)] / h$ for every right differentiable function $F$.

Then the operator $H$ is given by the following singular integral operator:

$$
H f(x)=\frac{1}{2} h(x)+\frac{1}{2 \pi i} \mathrm{P} . \mathrm{V} \cdot \int_{0}^{L} K\left(\alpha^{-1}(x), t\right) f(\alpha(t)) d t \quad(x \in \partial D) .
$$

We now recall the definition of Hardy spaces introduced by Fefferman and Stein:

For a function $f \in L^{1}\left(m_{j}\right)$, let $N(f)$ be the non-tangential maximal function of $f$, that is,

$$
N(f)(x)=\sup \{|\operatorname{PI}[f](z)|: z \in \Gamma(x)\}, \quad x \in \partial D,
$$

where $\Gamma(x)=\{z \in D:|z-x|<2 \operatorname{dist}(z, \partial D)\}$.

Fefferman and Stein's spaces are defined in terms of $N(\cdot)$ by:

$$
H_{\max }^{p}\left(m_{j}\right)=\left\{f \in L^{1}\left(m_{j}\right):\|f\|_{p, j, \max } \equiv\|N(f)\|_{p, j}<\infty\right\},
$$

where $\|\cdot\|_{p, j}$ is the $L^{p}\left(m_{j}\right)$-norm, $j=0,1,2$.

It is well known that $H_{\max }^{1}\left(m_{j}\right)$ is a proper subspace of $L^{1}\left(m_{j}\right)$ and $H_{\max }^{p}\left(m_{j}\right)=L^{p}\left(m_{j}\right), 1<p<\infty$ (see [5] and [8]).

In this paper we denote by $P(z, x)(z \in D, x \in \partial D)$ the Poisson kernel 
of $D$, and we put $W_{j}(t)=P\left(z_{j}, \alpha(t)\right)$, where $z_{j}$ is the point such that $m_{j}(F)=\int_{F} P\left(z_{j}, x\right) d m_{0}(x)$ for every Borel set $F$ of $\partial D, j=1,2$.

Our main theorem is the following:

THEOREM 1. Let $D$ be a domain bounded by finitely many non-intersecting simple closed $C^{4}$ regular curves. Let

$$
a_{j}(s, t)=-K(t, s)^{-} \cdot W_{j}(t) W_{j}(s)^{-1}-K(s, t),
$$

for $(s, t) \in[0, L) \times[0, L)-\{$ diagonal $\}, j=0,1,2$, where $W_{0}(t) \equiv 1$, and the bar denotes the complex conjugation here and elsewhere. Then we have the following:

(1) Each $a_{j}$ can be extended to a function $A_{j}$ on $[0, L) \times[0, L)$ in such a way that $A_{j}\left(\alpha^{-1}(\cdot), \alpha^{-1}(\cdot)\right)$ is continuous on $\partial D \times \partial D$.

(2) Let

$$
A_{j} f(x)=\frac{1}{2 \pi i} \int_{0}^{L} A_{j}\left(\alpha^{-1}(x), t\right) f(\alpha(t)) d t, \quad f \in L^{1}\left(m_{l}\right),
$$

$j, l=0,1,2$. Then each mapping $I-A_{j}$ is a bijective bounded operator of $H_{\mathrm{max}}^{1}\left(m_{l}\right)$ to $H_{\max }^{1}\left(m_{l}\right), j, l=0,1,2$.

Hence $\left(I-A_{j}\right)^{-1}$ is also bounded on $H_{\max }^{1}\left(m_{l}\right), j, l=0,1,2$. Moreover, for every $f \in L^{2}\left(m_{l}\right), l=0,1,2$, we have

$$
P_{j} f=H\left(I-A_{j}\right)^{-1} f, \quad j=0,1,2,
$$

and

$$
P_{j} f=P_{j+1}\left(I-A_{j+1}\right)\left(I-A_{j}\right)^{-1} f, \quad j=0,1 .
$$

As a consequence of Theorem 1 we have the following:

Corollary 1. Let $D$ be as in Theorem 1 . For every $j \in\{0,1,2\}$, the following are equivalent:

(1) $\|H f\|_{1, j} \leqq C_{1}\|f\|_{1, j, \max }$ for every $f \in L^{2}\left(m_{j}\right)$.

(2) $\left\|P_{0} f\right\|_{1, j} \leqq C_{2}\|f\|_{1, j, \max }$ for every $f \in L^{2}\left(m_{j}\right)$.

(3) $\left\|P_{1} f\right\|_{1, j} \leqq C_{3}\|f\|_{1, j, \max }$ for every $f \in L^{2}\left(m_{j}\right)$.

(4) $\left\|P_{2} f\right\|_{1, j} \leqq C_{4}\|f\|_{1, j, \max }$ for every $f \in L^{2}\left(m_{j}\right)$.

Here $C_{1}, C_{2}, C_{3}$ and $C_{4}$ are constants independent of $f$.

From the atomic decomposition of $H_{\max }^{1}\left(m_{j}\right)$ (cf. [8]) and a result in Coifman and Weiss [4, p. 559] it follows that the map $H$ can be extended to a bounded operator of $H_{\max }^{1}\left(m_{j}\right)$ to $L^{1}\left(m_{j}\right)$. Hence by Corollary 1 we have the following:

Corollary 2. Let $D$ be as in Theorem 1. Then the operator $H$ and the orthogonal projections $P_{j}$ can be extended to bounded projections of 
$H_{\max }^{1}\left(m_{l}\right)$ onto $H^{1}\left(m_{l}\right), j, l=0,1,2$.

When $D$ is the open unit disc and $m_{0}=m_{1}=m_{2}$, then this corollary was obtained by Burkholder, Gundy and Silverstein [2].

The following result is an immediate consequence of Corollary 2 and the $H_{\max }^{1}-B M O$ duality theorem. For the definition of $B M O$, see Section 2.

Corollary 3. Let $D$ be as in Theorem 1. The dual of $H^{1}\left(m_{j}\right)$ is isomorphic to $B M O A\left(m_{j}\right)$, where $B M O A\left(m_{j}\right)=B M O\left(m_{j}\right) \cap H^{2}\left(m_{j}\right), j=0,1,2$.

In connection with M. Riesz's inequality, Gamelin and Lumer [6] proved the following formula in an abstract setting:

$$
L^{p}\left(m_{1}\right)=H^{p}\left(m_{1}\right) \oplus H_{0}^{p}\left(m_{1}\right)^{-} \oplus N, \quad 1<p<\infty,
$$

where $H_{0}^{p}\left(m_{1}\right)=\left\{f \in H^{p}\left(m_{1}\right): \int f d m_{1}=0\right\}$ and $N$ is a finite dimensional subspace of $L^{\infty}\left(m_{1}\right)$.

As an application of Corollary 2 we can extend the result to the case $p=1$ as follows:

Corollary 4. Let $D$ be as in Theorem 1. Then

$$
H_{\max }^{1}\left(m_{1}\right)=H^{1}\left(m_{1}\right) \oplus H_{0}^{1}\left(m_{1}\right)^{-} \oplus N \text {. }
$$

In Section 2, we obtain propositions which will be used for the proofs of these results, and in Section 3 we prove Theorem 1. Corollaries stated above are proved in Section 4 .

2. Some preliminary results. Let $D$ be as in Theorem 1 . We will use $C_{5}, C_{6}, \cdots$ to denote positive constants depending only on $D, m_{1}$ and $m_{2}$.

Proposition 1 (cf. [12]). For every $j=1,2$ and every measurable set $E$, the following inequalities are valid:

$$
C_{5} m_{j-1}(E) \leqq m_{j}(E) \leqq C_{8} m_{j-1}(E) \text {. }
$$

Denote by $B M O\left(m_{j}\right)$ the class of all integrable functions $f$ such that $\|f\|_{b m o, j}=\sup \left\{\left(1 / m_{j}(I)\right) \int_{I}\left|f-f_{I}\right| d m_{j}: I\right.$ is the intersection of $\partial D$ and a disc centered at a point in $\partial D\}<\infty$. Here $f_{I}=m_{j}(I)^{-1} \int_{I} f d m_{j}$.

Proposition 2 ( $H_{\max }^{1}-B M O$ duality; see [8]). The dual of $H_{\max }^{1}\left(m_{j}\right)$ is isomorphic to $B M O\left(m_{j}\right)$. Especially, for every $x^{*} \in\left(H_{\max }^{1}\left(m_{j}\right)\right)^{*}$, there exists a unique element $b\left(x^{*}\right)$ of $B M O\left(m_{j}\right)$ such that

$$
x^{*}(f)=\int f \cdot b\left(x^{*}\right)^{-} d m_{j}, \text { for all } f \in L^{2}\left(m_{j}\right) .
$$


To prove Theorem 1, we need the following:

Proposition 3. (1) (cf. [9] and Proposition 1). H is a bounded operator of $L^{2}\left(m_{j}\right)$ to $L^{2}\left(m_{j}\right), j=0,1,2$.

(2) Furthermore, $H$ is a bounded projection of $L^{2}\left(m_{j}\right)$ onto $H^{2}\left(m_{j}\right)$, $j=0,1,2$.

Proof of (2). We note that the Cauchy integral

$$
F(z)=\frac{1}{2 \pi i} \int_{\partial D} \frac{1}{\zeta-z} f(\zeta) d \zeta \quad(z \in D)
$$

of a smooth function $f$ is Holder continuous near the boundary of $D$ (see [11]).

To prove $H H=H$, we recall Mergelyan's theorem which asserts that every $f \in A(\partial D)$ is approximated uniformly by rational functions whose poles are off $D$ (see [13]). For such rational functions $g$ we have $H g=g$ by the Cauchy integral formula. Hence by Propositions 3, (1) and the boundary property of the Cauchy integral stated above, we have

(i) $H g=g$, for every $g \in H^{2}\left(m_{j}\right)$, and

(ii) $H g \in A(\partial D)$, for every $g \in C^{2}(\partial D)$.

From (i) and (ii) it follows that $H$ is a bounded projection of $L^{2}\left(m_{j}\right)$.

By Proposition 3, (1), a density argument and the relation (i), we see immediately that the range of $H$ coincides with $H^{2}\left(m_{j}\right)$.

In order to prove the corollaries we need the following:

Proposition 4. If $f \in H^{1}\left(m_{j}\right)$ then

$$
\|f\|_{1, j} \leqq\|f\|_{1, j, \max } \leqq C_{7}\|f\|_{1, j} .
$$

Proof. Since the first inequality is clear, we prove the second. Here we use the following lemma which extends a result of Burkholder, Gundy and Silverstein [2] to certain general domains:

LEMMA 1 (cf. [1]). Let $\{B(t): 0 \leqq t<\infty\}$ be a complex Brownian motion starting at $z_{1}$. Then

$$
E\left[\sup _{0 \leq t<T}|\mathrm{PI}[f](B(t))|\right] \leqq C_{8}\|f\|_{1,1, \max },
$$

for every $f \in H_{\max }^{1}\left(m_{1}\right)$, and

$$
C_{\vartheta}\|f\|_{1,1, \max } \leqq E\left[\sup _{0 \leq t<T}|\mathrm{PI}[f](B(t))|\right],
$$

for every $f \in L^{1}\left(m_{1}\right)$ with $E\left[\sup _{0 \leq t<T}|\operatorname{PI}[f](B(t))|\right]<\infty$, where $E$ is the expectation with respect to the Wiener measure which defines the Brownian motion $B$, and $T$ is the first time at which $B$ escapes from $D$. 
This lemma holds good if $D$ is higher dimensional non-tangentially accessible domains (see [1]).

Now we proceed with the proof of Proposition 4. Let $M f(t)=$ $\mathrm{PI}[f](B(t))$ for $0 \leqq t<T$, and $M f(t)=f(B(T))$ for $T \leqq t$. By Ito's formula, $M f(t)$ is a continuous holomorphic martingale in the sense of Varopoulos [15]. Consequently, by [15], $|M f(t)|^{1 / 2}$ is a submartingale. Hence Doob's inequality implies that

$$
E\left[\sup _{0 \leq t<T}|\mathrm{PI}[f](B(t))|\right]=E\left[\left(\sup _{0 \leq t \leq \infty}|M f(t)|^{1 / 2}\right)^{2}\right] \leqq 4 E[|M f(\infty)|]=4\|f\|_{1,1} .
$$

Here the last equality is guranteed by Kakutani's theorem. Thus by Proposition 1 we obtain Proposition 4.

3. Proof of Theorem 1. Proof of (1). Let

$$
\begin{aligned}
& q(s, t)=-K(t, s)^{-}-K(s, t) \text { and } \\
& r_{j}(s, t)=W_{j}(s)^{-1}\left(W_{j}(s)-W_{j}(t)\right)\left(K(t, s)^{-}\right) .
\end{aligned}
$$

Then $a_{j}=q+r_{j}$.

By the proof of Theorem 1 in Kerzman and Stein [10], $q$ is the restriction of a function which is continuous on each $I_{k} \times I_{m}(k, m=0, \cdots, n)$. Hence it is sufficient to prove that $r_{j}$ can be extended to a function which is continuous on each $I_{k} \times I_{m}(k, m=0, \cdots, n)$. To prove this we show the following:

LEMma 2. $W_{j}$ is twice continuously differentiable in each $I_{k}(k=$ $0, \cdots, n), j=0,1,2$.

Proof. Let

$$
n(t)=-i D_{+} \alpha(t),
$$

For sufficiently small $\varepsilon>0$, let $\alpha(\varepsilon, t)=\alpha(t)-\varepsilon n(t)$. It is easy to check that $\Gamma_{k, \varepsilon} \equiv\left\{\alpha(\varepsilon, t): t \in I_{k}\right\}$ is a simple closed curve and $\Gamma_{0, \varepsilon}+\cdots+\Gamma_{n, \varepsilon}$ is the boundary of a $C^{3}$ subdomain $D_{\varepsilon}$ of $D$ such that $D_{\varepsilon} \uparrow D$ as $\varepsilon \rightarrow 0+$.

Let $n(\varepsilon, t)$ be the outward normal field of $\partial D_{\varepsilon}$, that is,

$$
n(\varepsilon, t)=\left(1 /\left|D_{+} \alpha(\varepsilon, t)\right|\right)\left(\operatorname{Im} D_{+} \alpha(\varepsilon, t) \frac{\partial}{\partial x}-i \operatorname{Re} D_{+} \alpha(\varepsilon, t) \frac{\partial}{\partial y}\right),
$$

where $D_{+} \alpha(\varepsilon, t)=\lim _{h \rightarrow 0+}[\alpha(\varepsilon, t+h)-\alpha(\varepsilon, t)] / h$.

By regularity properties of elliptic boundary value problems ([7]), the Green function $g\left(z_{j}, \cdot\right)$ of $D$ possesses all derivatives of order $\leqq 3$ continuous in $D \backslash\left\{z_{j}\right\}$ and they have continuous extensions to $D \cup \partial D \backslash\left\{z_{j}\right\}$. Hence if we put

$$
W_{j, \varepsilon}(t)=-n(\varepsilon, t) g\left(z_{j}, \alpha(\varepsilon, t)\right),
$$


then $W_{j, \varepsilon} \in C^{2}\left(I_{k}\right)$, and $W_{j, \varepsilon}$ converges to the Poisson kernel $W_{j}$ in the $C^{2}\left(I_{k}\right)-$ topology as $\varepsilon \rightarrow 0+(k=0, \cdots, n)$. Consequently, $W_{j} \in C^{2}\left(I_{k}\right) k=0, \cdots, n$.

Now, we return to the proof of Theorem 1. By Lemma 2 it suffices to show that $\left(W_{j}(s)-W_{j}(t)\right) K(t, s)$ is the restriction of a function which is continuous on each $I_{k} \times I_{m}(k, m=0, \cdots, n)$. Let

and

$$
F_{j}(s, t)= \begin{cases}{\left[W_{j}(s)-W_{j}(t)\right] /(s-t)} & \text { if } s \neq t \\ D_{+} W_{j}(t) & \text { if } \quad s=t\end{cases}
$$

$$
G(s, t)= \begin{cases}{[\alpha(s)-\alpha(t)] /(s-t)} & \text { if } s \neq t \\ D_{+} \alpha(t) & \text { if } s=t .\end{cases}
$$

Then $\left(W_{j}(s)-W_{j}(t)\right) K(t, s)=D_{+} \alpha(s) \cdot F_{j}(s, t) / G(s, t)$ for every $(s, t) \epsilon$ $[0, L) \times[0, L)-\{$ diagonal $\}$. By the Taylor expansion of $W_{j}(s)-W_{j}(t)$ we see that

$$
F_{j}(t+u, t+v)=D_{+} W_{j}(t)+o(1)
$$

if $u \neq v$ and if $t, t+u$ and $t+v$ belong to a same interval $I_{k}$. Moreover, $F_{j}$ is continuous on each $\left(I_{k} \times I_{m}\right) \cap$ \{diagonal $\}$ by definition. Hence $F_{j}$ is continuous on each $I_{k} \times I_{m}$. A similar proof yields that $G \in C\left(I_{k} \times I_{m}\right)$ and $G \neq 0, k, m=0, \cdots, n$. This complete the proof of (1).

Proof of Theorem 1, (2). By Proposition 1 it is sufficient to show (2) when $j=l$.

For a compact operator $T$ on a Banach space the mapping $I-T$ is a Fredholm operator of index zero ([14, p. 301]). Hence (2) is valid if the following assertions hold true:

Assertion 1. $A_{j}$ is compact on $H_{\max }^{1}\left(m_{j}\right)$.

Assertion 2. The range of $I-A_{j}$ is equal to $H_{\max }^{1}\left(m_{j}\right)$.

We begin by proving Assertion 1. By Theorem 1, (1) and the generalized Stone-Weierstrass theorem ([13, Corollary 12.5]) there exist $p_{j, \nu} \in$ $\left\{\sum_{m=1}^{N} b_{m}(x) c_{m}(y): b_{m} \in C(\partial D), c_{m} \in C(\partial D), N=1,2, \cdots\right\}$ such that

$$
\lim _{\nu \rightarrow \infty}\left\|A_{j}\left(\alpha^{-1}(\cdot), \alpha^{-1}(\cdot)\right)-p_{j, \nu}\right\|_{\infty}=0 .
$$

Since the integral operators

$$
P_{j, \nu} f(x)=\int p_{j, \nu}(x, \alpha(t)) f(\alpha(t)) d t
$$

are of finite rank, we obtain Assertion 1 by Hölder's inequality.

Before verifying Assertion 2, we introduce some notation:

For a Banach space $X$, the algebra of all bounded linear operators from $X$ to itself will be denoted by $\mathrm{BL}(X)$. If $T \in \mathrm{BL}\left(H_{\max }^{1}\left(m_{j}\right)\right)$, then 
$(T)_{m}$ denotes the conjugate operator of $T$ as an operator on $H_{\max }^{1}\left(m_{j}\right)$. If $T \in \mathrm{BL}\left(L^{2}\left(m_{j}\right)\right)$, then $(T)_{L}$ represents the adjoint as an element of $\mathrm{BL}\left(L^{2}\left(\mathrm{~m}_{j}\right)\right)$. For every $T \in \mathrm{BL}\left(H_{\max }^{1}\left(m_{j}\right)\right)$, we put $R(T)=\left\{T f: f \in H_{\max }^{1}\left(m_{j}\right)\right\}$.

By the closed range theorem and [14, V. Theorem 7.8] we have

$$
\begin{aligned}
R\left(I-A_{j}\right)= & \left\{g \in H_{\max }^{1}\left(m_{j}\right): g^{*}(g)=0 \text { for every element } g^{*}\right. \text { of the kernel } \\
& \text { of } \left.\left(I-A_{j}\right)_{m}\right\} .
\end{aligned}
$$

Hence, to prove Assertion 2 we need only to show that the kernel $K$ of $\left(I-A_{j}\right)_{m}$ consists of zero.

LEMmA 3. For every $g^{*} \in\left(H_{\max }^{1}\left(m_{j}\right)\right)^{*}$ and every $g \in L^{2}\left(m_{j}\right)$,

$$
\left[\left(A_{j}\right)_{m}\left(g^{*}\right)\right](g)=\int g \cdot H\left(b\left(g^{*}\right)\right)^{-} d m_{j}-\int g \cdot(H)_{L}\left(b\left(g^{*}\right)\right)^{-} d m_{j},
$$

where $b\left(g^{*}\right)$ is defined as in Proposition 2.

Proof of Lemma 3. It is easy to check that

$$
(H)_{L}(h)(x)=\frac{1}{2} h(x)-\frac{1}{2 \pi i} \mathrm{P} . \mathrm{V} \cdot \int K\left(t, \alpha^{-1}(x)\right)^{-} h(\alpha(t)) W_{j}(t) W_{j}\left(\alpha^{-1}(x)\right)^{-1} d t
$$

and $A_{j}(h)=(H)_{L}(h)-H(h)$, for every $h \in L^{2}\left(m_{j}\right)$. Hence by Proposition 2 we have

$$
\begin{aligned}
{\left[\left(A_{j}\right)_{m}\left(g^{*}\right)\right](g) } & =\int(H)_{L}(g) \cdot b\left(g^{*}\right)^{-} d m_{j}-\int H(g) \cdot b\left(g^{*}\right)^{-} d m_{j} \\
& =\int g \cdot H\left(b\left(g^{*}\right)\right)^{-} d m_{j}-\int g \cdot(H)_{L}\left(b\left(g^{*}\right)\right)^{-} d m_{j},
\end{aligned}
$$

which prove Lemma 3.

Now we are ready to prove that $K=\{0\}$. Fix any $g^{*} \in K$. Then for every $g \in L^{2}\left(m_{j}\right)$ we obtain

$$
\begin{aligned}
0 & =\left[\left(I-A_{j}\right)_{m}\left(g^{*}\right)\right](g) \\
& =g^{*}(g)-\int g \cdot H\left(b\left(g^{*}\right)\right)^{-} d m_{j}+\int g \cdot(H)_{L}\left(b\left(g^{*}\right)\right)^{-} d m_{j} \\
& =\int g \cdot\left[I-H+(H)_{L}\right]\left(b\left(g^{*}\right)\right)^{-} d m_{j} .
\end{aligned}
$$

Hence $b\left(g^{*}\right)=\left[H-(H)_{L}\right]\left(b\left(g^{*}\right)\right)$. The last relation implies that

$$
\begin{aligned}
\left\|b\left(g^{*}\right)\right\|_{2, j}^{2} & =\int H\left(b\left(g^{*}\right)\right) \cdot b\left(g^{*}\right)^{-} d m_{j}-\int(H)_{L}\left(b\left(g^{*}\right)\right) \cdot b\left(g^{*}\right)^{-} d m_{j} \\
& =2 i \operatorname{Im}\left[\int H\left(b\left(g^{*}\right)\right) \cdot b\left(g^{*}\right)^{-} d m_{j}\right] .
\end{aligned}
$$

Consequently, $\left\|b\left(g^{*}\right)\right\|_{2, j}=0$. Thus $K=\{0\}$. 
Proof of Theorem 1, (3) and (4). We have $P_{j} H=H$ and $P_{j}(H)_{L}=P_{j}$, because $\left(P_{j}\right)_{L}=P_{j}$. Hence $P_{j}\left(I+H-(H)_{L}\right)=H$. By Theorem 1, (2), $I-A_{j}$ is an injective bounded operator of $L^{2}\left(m_{j}\right)$ to $L^{2}\left(m_{j}\right)$. Furthermore $I-A_{j}$ is a Fredholm operator on $L^{2}\left(m_{j}\right)$ of index zero. Therefore by the proof of Lemma 3 we obtain (3). From (3) follows

$$
P_{j}=H\left(I-A_{j}\right)^{-1}=P_{j+1}\left(I-A_{j+1}\right)\left(I-A_{j}\right)^{-1},
$$

which completes our proof.

4. Proofs of corollaries. Corollary 1 is proved directly by Theorem 1 .

Corollary 2 is an immediate consequence of Corollary 1, Proposition 3, (2) and Proposition 4, because the operator $H$ is a bounded map of $H_{\max }^{1}\left(m_{j}\right)$ to $L^{1}\left(m_{j}\right)$ as mentioned in Section 1 . Corollary 3 is proved easily by Corollary 2 and a usual argument.

Proof of Corollary 4. Corollary 2 implies that

$$
H_{\max }^{1}\left(m_{1}\right)=H^{1}\left(m_{1}\right) \oplus\left[I-P_{1}\right]\left(H_{\max }^{1}\left(m_{1}\right)\right) \text {. }
$$

Let $Z=H_{0}^{1}\left(m_{1}\right)^{-} \bigoplus N$, where $N$ is a finite dimensional subspace of $L^{\infty}\left(m_{1}\right)$ defined by Gamelin and Lumer [6]. We show that $\left[I-P_{1}\right]\left(H_{\max }^{1}\left(m_{1}\right)\right)=Z$.

The space $Z$ is closed in $H_{\max }^{1}\left(m_{1}\right)$, because $N$ is finite dimensional. Hence applying the open mapping theorem to the operator $T(g, h)=g+h$ $\left((g, h) \in H_{0}^{1}\left(m_{1}\right)^{-} \times N\right)$, we have

$$
C\left(\|g\|_{1,1, \max }+\|h\|_{1,1, \max }\right) \leqq\|g+h\|_{1,1, \max } \leqq\|g\|_{1,1, \max }+\|h\|_{1,1, \max },
$$

where $(g, h) \in H_{0}^{1}\left(m_{1}\right)^{-} \times N$ and $C$ is a constant independent of $g$ and $h$. From Corollary 2 follows

$$
\left\langle\left[I-P_{1}\right]\left(L^{2}\left(m_{1}\right)\right)\right\rangle=\left[I-P_{1}\right]\left(H_{\max }^{1}\left(m_{1}\right)\right),
$$

where $\langle U\rangle$ denotes the $H_{\max }^{1}\left(m_{1}\right)$-norm closure of $U$. Furthermore from Proposition 4 follow

Since

$$
\left\langle H_{2}^{0}\left(m_{1}\right)^{-}\right\rangle=H_{0}^{1}\left(m_{1}\right)^{-} \text {and }\langle N\rangle=N .
$$

$$
L^{2}\left(m_{1}\right)=H^{2}\left(m_{1}\right) \oplus H_{0}^{2}\left(m_{1}\right)-\oplus N,
$$

(cf. [6]), we have by (i)

$$
\left[I-P_{1}\right]\left(H_{\max }^{1}\left(m_{1}\right)\right)=\left\langle H_{0}^{2}\left(m_{1}\right)^{-} \oplus N\right\rangle=\left\langle H_{0}^{2}\left(m_{1}\right)^{-}\right\rangle \bigoplus\langle N\rangle=H_{0}^{1}\left(m_{1}\right)^{-} \oplus N,
$$

from which Corollary 4 follows.

\section{REFERENCES}

[1] H. AraI, Brownian motion and the Hardy space $H^{1}$ on non-tangentially accessible domains, Sci. Res. School of Education, Waseda Univ. 34 (1985), 21-29. 
[2] D. L. Burkholder, R. F. Gundy AND M.L. Silverstein, A maximal function characterization of the class $H^{p}$, Trans. Amer. Math. Soc. 157 (1972), 137-153.

[3] J. Burbea, The Cauchy and Szegö kernels on multiply connected regions, Rend. Circ. Mat. Palermo, (2) 31, (1982), 105-118.

[4] R. R. CoIfman and G. Weiss, Extensions of Hardy spaces and their use in analysis, Bull. Amer. Math. Soc. 83 (1977), 569-645.

[5] C. Feffierman ANd E. M. Stein, $H^{p}$ spaces of several variables, Acta Math. 129 (1972), 137-193.

[6] T. Gamelin and G. Lumer, Theory of abstract Hardy spaces and the universal Hardy class, Adv. in Math. 2, (1968), 118-174.

[7] D. Gilberg and N. S. Trudinger, Elliptic Partial Differential Equations of Second Order, Springer Verlag, Berlin, Heidelberg, New York, Tokyo, 1983.

[8] D. S. JERISON AND C. E. KENIG, Boundary behavior of harmonic functions in non-tangentially accessible domains, Adv. in Math. 46, (1982), 81-147.

[9] J. L. Journé, Calderón-Zygmund Operators, Pseudo-Differential Operators and the Cauchy integral of Calderón, Lecture Notes in Math. 994, Springer Verlag, Berlin, Heidelberg, New York, Tokyo, 1983.

[10] N. Kerzman and E. M. Stein, The Cauchy kernel, the Szegö kernel, and the Riemann mapping function, Math. Ann. 236 (1978), 85-93.

[11] M. I. MuskHeLISHVili, Singular Integral Equations, P. Nordhoff, Groningen, The Netherlands, 1953.

[12] E. M. Stein, Boundary Behavior of Holomorphic Functions in Several Variables, Princeton Univ. Press 1972.

[13] E. L. Sтоuт, The Theory of Uniform Algebras, Bogden and Quigley, Belmont, California, 1971.

[14] A. E. TaYlor and D. C. LaY, Introduction to Functional Analysis, John Wiley \& Sons, New York, Chichester, Brisbane, Toronto, 1980.

[15] N. Th. Varopoulos, The Helson-Szegö theorem and $A_{p}$ functions for Brownian motion and several variables, J. Funct. Analysis, 39 (1980), 85-121.

Mathematical Institute

TÔHOKU UNIVERSITY

SENDAI 980

JAPAN 